\title{
Contextual processing of electrical capacitance tomography measurement data for temporal modeling of pneumatic conveying process.
}

\author{
Andrzej Romanowski \\ Institute of Applied Computer Science \\ Lodz University of Technology \\ Lodz, 90-924 Poland \\ Email: androm@iis.p.lodz.pl
}

\begin{abstract}
This work covers deployment of contextual processing of measurement data in application to temporal modeling of pneumatic conveying industrial process. Electrical capacitance tomography (ECT) used as a non-invasive process monitoring tool is supported by data mining for regularization of nonlinear inverse problem solution. Processing of a larger number of archived experimental datasets enables extracting additional constraints for inference. Contextual data processing model (CDPM) extracts demanded information from the data in order to incorporate it as an expert knowledge about the process temporal behavior. Then it is incorporated into the Bayesian inference framework. Comparative analysis with previous work and domain expert prepared baseline to the proposed approach is demonstrated. Additionally, simplified parameterization is tested and verified by the quantitative experimental analysis.
\end{abstract}

\section{INTRODUCTION AND RELATED WORK}

\section{A. Pneumatic conveying and ECT}

Bulk solids, powders and particulates cover about $2 / 3$ of all solid materials used in industry at various stages of manufacturing. However, proper monitoring of processes that involve bulk solids is difficult because of their volumetric and opaque nature. The most promising techniques involve non-invasive and non-intrusive tools such process tomography methods, while electric capacitance tomography is one of the most popular modalities [1] [2]. However, there are some issues related to nonlinear nature of electrical field associated with extracting required process-related information from ECT data [3] [4] [5]. Therefore some methods aiming at improving the inverse problem conditions were developed over last 20 years [6] [7] [8] [9] [10] [11] [12] [13] [14]. Here a contextual tomography-based measurement data processing approach is proposed. It is based on the same contextual data processing model (CDPM) as described in [15] that was valided for big data driven aspects there. In contrast, current work validates CDPM within the scope of inverse problem regularization support. The main contribution of this work is therefore the proposed theoretical model for contextual data processing applied to temporal inference about the process behaviour. It is validated here with binary classification technique comparing to the baseline Bayesian inference framework.

\section{B. Contextual Model for Measurement Data Processing}

Contextual methods are derived from a concept of contextaware services or context-awareness in general. These concepts are extensively used in human computer interaction (HCI) discipline. Here it is postulated to outspread these notions to the field of measurement data processing for industrial processes monitoring [16] [17] [18]. Though some modification is required but the core of the concept remains to be based on a simple idea of using the additionally available information describing the object of interest in order to broaden the set of data to be incorporated as the input to the system.

I propose to expand this typical understanding of context

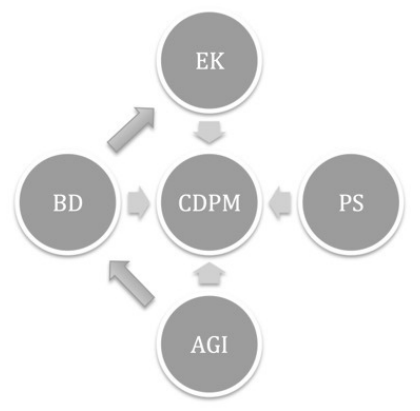

Fig. 1: General diagram of contextual data processing model (CDPM). EK, BD AGI, PS refer to as Expert Knowledge, Big Data, Artificially Generated Input, Peripheral Sensors accordingly.

related to the information coming from peripheral sensors (PS) to the four inter-related categories that form the CDPM model as shown on Fig.1. While PS still stays as substantial pillar of the model, the extension goes towards incorporating the following factors: Expert knowledge (EK), Big Data analysis (BD) and Artificially generated inputs (AGI) into the model. EK refers to any prior knowledge that can be defined independently to the current situation (experiment). BD stands for a broader base of previously conducted experiments or measurement datasets that can be analyzed in order to search for similarities, patterns or knowledge that can be extracted 
out of it. AGI works as a complementary tool to supplement knowledge base, especially in case of sporadic phenomena for events that are rarely captured. While AGI can be either a distinct pillar of the CDPM it can also contribute to BD component. While exploring the BD analysis for CDPM is postulated in [15] [19] [20], this paper focuses at showing the method for incorporating EK into the process of inverse problem solving for ECT application to the monitoring of pneumatic conveying flow of bulk solids.

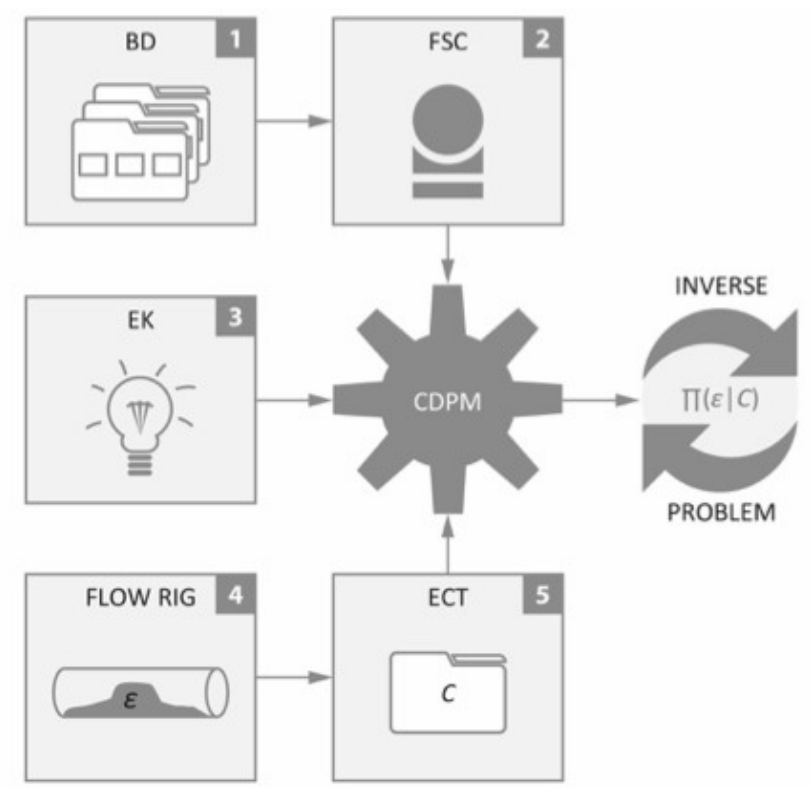

Fig. 2: CDPM for temporal modeling of pneumatic conveying. EK, BD, PS, FSC, ECT, C refer to as Expert Knowledge, Big Data, Artificially Generated Input, Peripheral Sensors, Flow State Classifiers, Electrical Capacitance Tomography, Capacitance data accordingly.

\section{Pneumatic Conveying Experimental Setup}

Experimental part of this research was conducted at the Tom Dyakowski Process Tomography Laboratory at the Lodz University of Technology. The ECT dual plane, 8-electrode sensors were fixed on a $65 \mathrm{~mm}$ horizontal section of pneumatic conveying test rig as shown on Fig. 2. More details about the equipment can be found here [18]. Measurement campaign spanned over a range of settings preserving regular slug flow for different combinations of material feed rate, and air pressure $(10.0-16 \mathrm{~Hz}$ inverter, $60-100 \mathrm{~Hz}$ of the rotary valve) for approx. 3 cubic mm polyamide pellets.

\section{INVESTIGATION PROCEDURE}

\section{A. Baseline requirements}

This work aims at proving that using available previous experimental data one can derive information useful for current measurement-related computational problem [10]. Spatiotemporal modeling of pneumatic conveying based on Bayesian inference and statistical methods demonstrated possibility of

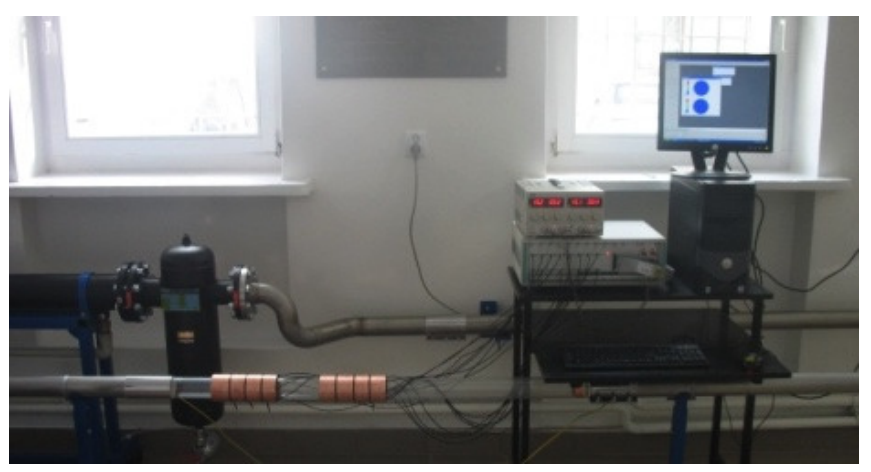

Fig. 3: Experimental setup: ECT sensor equipped measurement section at horizontal pneumatic conveying rig and a corresponding ECT data acquisition device.

omitting the image reconstruction stage on the way to estimate characteristic flow parameters [8]. Current step is to simplify parametric modeling within the temporal modeling concurrently preserving or increasing the accuracy with the aid of CDPM. Computational environment with use of Hadoop is similar to desribed in [21]. The investigation is based upon the following postulations:

1) Mean concentration of bulk solid is taken as a main parameter describing flow state at any time point. ECT is the main measurement tool to supply estimated electric permittivity distribution (related to bulk concentration) based on capacitance measurement vectors.

2) There is additional information available in form of archived experimental datasets coupled with supplementary information such as estimated flow rate and weighed quantity of total material being transported, material geometry, properties, valves states, other metadata. Fig. 2 illustrates the basic workflow for the inverse problem using CPDM support.

3) Fragments of archive datasets are taken especially for slug rise (ECT recorded slug build up) and fall (ECT recorded slug tail) in order to regularize inverse problem for temporal analysis. Previously geometrical modeling was proposed for temporal smoothing varied in time that resulted in high uncertainty [22].

4) CPDM takes fragments, full-lenght experimental datasets and optional classifiers built on top of BD employment as well as any other general knowledge in order to incorporate it into the EK inverse problem solution as described in [8].

5) Correspondence to baseline, expert-annotated datasets in terms of mean electrical permittivity change in time was proposed as a principal measure to assess the proposed approach accuracy.

6) Calculated total transported material weight (relevant to flow rate) was chosen as an extra measure to verify if the simplification relying on substitution of geometrical modeling with mean concentration change is reasonable. 


\section{B. Inference Framework}

Eq. (1) shows the approximation of the posterior probability density function related to the unknown distribution of the electric permittivity of the transported bulk solids mixture within the ECT sensor space. The critical factor from the CDPM is the $k E$ which stands for the prior knowledge with relation to the expected constraints on the electric permittivity $\epsilon$ distribution in relation to obtained electric capacitance records $\mathrm{C}$ and in fact $\mathrm{kE}(\epsilon)$ is $\mathrm{EK}$ equivalent.

$$
p(\varepsilon \mid C) \propto p(C \mid \varepsilon) * k_{E}(\varepsilon)
$$

where $\mathrm{p}(\epsilon)$ is the inverse problem for ECT and bulk solids flow and the $\mathrm{p}(\mathrm{C}-\epsilon)$ is the forward problem that can be numerically approximated using FEM method. Hence the EK can be defined here as the regularization prior, and for an electric permittivity case can be denoted as $\operatorname{kE}(\epsilon) \mathrm{Eq}(2)$ :

$$
k_{E}(\varepsilon) \propto \exp \left\{-\beta_{s}\|\varepsilon\|_{l}^{l}\right\} ; \beta_{s}>0,0 \leq l \leq 2 .
$$

Such stated $\operatorname{kE}(\epsilon)$ leads to Laplacian distribution with a 1 (l=1) norm and leads to Gaussian distribution for norm 2 $(\mathrm{l}=2)$. Now extending this approach to a temporal dependence analysis the following relation expresses how consecutive frames dependence can be described (Eq. 3):

$$
\Pi\left(\kappa^{t} \mid \kappa^{t+1}, \kappa^{t-1}\right) \propto \exp \left\{-\beta_{t}\left(\kappa^{t}-\bar{\kappa} t\right)^{2}\right\}
$$

where $\mathrm{Kt}$ is a set of estimated parameters at a given time point $t$, and $\beta \mathrm{t}$ decides on the level of correlation between these values in consecutive time points (in contrast to $\beta \mathrm{s}$ in a spatial distribution case in Eq. 2). The procedure of tackling the is shown on right hand-side of Fig. 1. There are several possible options for solving the Bayesian-based approach for ECT inverse problem solving. Related work referred to here is based on highly iterative MCMC scheme that is both computationally and time demanding [8] [10]. Current work was decided to use the same option yet thanks to GPU computing and reduced number of parameters the calculations are far less demanding and time consuming as shown in [11].

\section{RESULTS}

\section{A. Comparative Analysis}

The results are given for the modeling of the pneumatic conveying slug flow for several different flow configurations as discussed in experimental setup section. Results are divided into 3 classes with respect to flow rate, i.e. average transported medium rate over time. Table 1 provides the comparison between the compliance of estimated mean concentration of solids (corresponding to amount of medium transported) on the basis of comparison between the raw data, reconstructed images analysis, previous approach [18] and the CDPM model. The datasets are cut to the 100 frames series that always include both the slug and stationary layer portion (i.e. consecutive periods of frames with lower or higher mean concentration values). Each of the 3 categories: low flow rate (Table 1, row 1), medium flow rate (Table 1 , row 2) and high flow rate (Table 1, row 3) are arbitrarily divided into 3 consecutively rising classes based on the results and parameters of the performed experiments. Results in rows 1-3 indicate percentage compliance averaged over 10 different calculations and standard deviation both rounded to first decimal digit. Percentage is calculated based on binary classification of a consecutive measurement frame as either belonging or not belonging to a slug as shown in [9]. Row 4 reveals average error. Row 5 shows supplementary measure of total material transported weight comparing to the scale-recorded values for

\begin{tabular}{|c|c|c|c|c|c|c|c|c|c|}
\hline & \multirow{3}{*}{$\begin{array}{l}\text { Slug flow } \\
\text { rates } \\
\text { averaged } \\
\text { over time }\end{array}$} & \multicolumn{8}{|c|}{$\begin{array}{l}\text { Results: average classification compliance (accuracy) } \\
\text { comparing to the expert ground truth baseline }\end{array}$} \\
\hline & & \multicolumn{2}{|c|}{ Raw data } & \multicolumn{2}{|c|}{$\begin{array}{l}\text { Reconstructed } \\
\text { images }\end{array}$} & \multicolumn{2}{|c|}{$\begin{array}{l}\text { Previous } \\
\text { approach }\end{array}$} & \multicolumn{2}{|c|}{$C D P M$} \\
\hline & & {$[\%]$} & SD & {$[\%]$} & SD & {$[\%]$} & SD & {$[\%]$} & $\mathrm{SD}$ \\
\hline 1 & $\begin{array}{l}\text { Low flow } \\
\text { rate }\end{array}$ & 78.6 & 15.8 & 91.3 & 6.9 & 89.6 & 7.1 & 90.4 & 6.3 \\
\hline 2 & $\begin{array}{l}\text { Medium flow } \\
\text { rate }\end{array}$ & 81.4 & 13.6 & 78.4 & 16.2 & 86.9 & 8.0 & 92.3 & 3.8 \\
\hline 3 & $\begin{array}{l}\text { High flow } \\
\text { rate }\end{array}$ & 92.3 & 6.1 & 86.5 & 11.6 & 88.8 & 5.5 & 94.6 & 2.3 \\
\hline 4 & $\begin{array}{l}\text { Average } \\
\text { error }\end{array}$ & 15.9 & & 14.6 & & 11.6 & & 7.6 & \\
\hline 5 & $\begin{array}{c}\text { Total } \\
\text { weight }\end{array}$ & 89.2 & 7.7 & 83.7 & 10.3 & 88.6 & 4.1 & 91.5 & 3.2 \\
\hline
\end{tabular}
whole experimental datasets.

TABLE I: Pneumatic conveying CDPM results

\section{B. Discussion and directions for future work}

The proposed CDPM model using temporal information as an input for expert knowledge (EK) extension to the normal prior knowledge applied in Bayesian inference framework performs well comparing to the baseline, i.e. to the expert provided ground truth in form of marked test datasets. CDPM results for all three classes reached 90\%+ accuracy. CDPM performs better than other methods especially for medium and high flow rates for which both reconstructed images postprocessing as well as previous work based on geometrical parameterization for temporal modeling obtained the weakest scores. Interesting feature is that the reconstructed images based analysis outperform both compared methods for low flow rates while is giving worse results for more dynamic flow regimes while the other two gain more accuracy for higher flow rates. It will be interesting to verify performance of the proposed model for a truly large data sample, especially of mixed origins of different experimental installations [23] [24]. On the other hand, it is noticeable that CDPM beats direct estimation based on raw data records by a small difference yet with much lower variance as well.

Total weight of material transported by the pneumatic conveying flow rig shown in row 5 of Tab. 1 showed superior 
performance of CDPM model over the others however accuracy on $91.2 \%$ is not yet sufficient to treat ECT-based systems as a reliable, stand-alone, online monitoring tool for pneumatic conveying process. Nevertheless as stated in the beginning this measure proved that simplification of modeling process meant by the reducing the parameters number is feasible. Model that assumed mean material concentration value related to mean electric permittivity performed better than geometrical modeling of assumed cross-sectional areas of homogeneous material distribution.

More extensive computational study is required to derive more definitive conclusion about the performance of the CDPM model. Especially, further research work on the larger number of datasets in order to verify the range of applicability of this method to more general classes of applications for ECT monitoring of powder flows in vertical and inclined sections is needed. Next step is to construct a distributed computational environment for big experimental measurement data employing map-reduced paradigm in order to cope and test CDPM performance extensively. It would be interesting to see the ARbased study and track users what and how these professionals perceive the industrial environments to obtain a baseline for further development [15][25][26].

\section{SUMMARY}

This work illustrates experimental verification of the proposed CDPM model for temporal modeling of industrial pneumatic conveying process. The method is based on the incorporation of the extra expert knowledge as the regularization factor into the inverse problem solving for electrical capacitance tomography. As the initial results show, the methodology is suitable for temporal modeling of ECT-based monitored pneumatic conveying of bulk solids flow since it helps to identify the flow states (regimes) with similar or better accuracy than the state of the art methods. Calculated total quantity of material transported based on the proposed approach is of approximately $5-10 \%$ more accurate than the previous research shown. Hence the proposed simplified model based on mean material concentration value seems to be sufficient and in at least some aspects superior over previously reported geometrical parameterization for temporal bulk solid flow modeling.

\section{REFERENCES}

[1] M. S. Beck and R. A. Williams, "Process tomography: a european innovation and its applications," Measurement Science and Technology, vol. 7, no. 3, p. 215, 1996.

[2] W. Q. Yang, A. L. Stott, M. S. Beck, and C. G. Xie, "Development of capacitance tomographic imaging systems for oil pipeline measurements," Review of Scientific Instruments, vol. 66, no. 8, pp. 4326-4332, 1995.

[3] W. Fang, "Reconstruction of permittivity profile from boundary capacitance data," Appl. Math. Comput., vol. 177, pp. 178-188, June 2006.

[4] K. Grudzien, A. Romanowski, D. Sankowski, and R. A. Williams, "Gravitational granular flow dynamics study based on tomographic data processing," Particulate Science and Technology, vol. 26, no. 1, pp. 67$82,2007$.

[5] D. Wanta, J. Kryszyn, J. Buraczyk, and W. T. Smolik, "Www interface for an electrical capacitance tomography system," in 2018 International Interdisciplinary PhD Workshop (IIPhDW), pp. 344-347, May 2018.
[6] M. Soleimani and W. R. B. Lionheart, "Nonlinear image reconstruction for electrical capacitance tomography using experimental data," Measurement Science and Technology, vol. 16, no. 10, p. 1987, 2005.

[7] K. Grudzien, "Visualization system for large-scale silo flow monitoring based on ect technique," IEEE Sensors Journal, vol. 17, pp. 8242-8250, December 2017.

[8] A. Romanowski, K. Grudzien, R. Aykroyd, and R. Williams, "Advanced statistical analysis as a novel tool to pneumatic conveying monitoring and control strategy development," Particle \& Particle Systems Characterization, vol. 23, no. 34, pp. 289-296, 2006

[9] T. Rymarczyk, P. Tchorzewski, P. Adamkiewicz, K. Duda, and J. Szumowski, "Practical implementation of electrical tomography in a distributed system to examine the condition of objects," IEEE Sensors Journal, vol. 7, no. 1, pp. 11-16, 2017.

[10] K. Grudzien, A. Romanowski, and R. Williams, "Application of a bayesian approach to the tomographic analysis of hopper flow," Particle \& Particle Systems Characterization, vol. 22, no. 4, pp. 246-253, 2006.

[11] A. Kowalska, R. Banasiak, R. Wajman, A. Romanowski, and D. Sankowski, "Towards high precision electrical capacitance tomography multilayer sensor structure using $3 \mathrm{~d}$ modelling and $3 \mathrm{~d}$ printing method," in 2018 International Interdisciplinary PhD Workshop (IIPhDW), IEEE, pp. 238-243, 2018.

[12] R. Banasiak, R. Wajman, T. Jaworski, P. Fiderek, P. Kapusta, and D. Sankowski, "Two-phase flow regime three-dimensonal visualization using electrical capacitance tomography algorithms and software," Informatyka, Automatyka, Pomiary w Gospodarce i Ochronie rodowiska, vol. T. 7, nr 1, pp. 11-16, 2017.

[13] M. Panczyk, T. Rymarczyk, and J. Sikora, "Comparison of the inverse problem solutions for a $2 \mathrm{~d}$ damp wall multilayer and nonhomogenous models," in 2018 International Interdisciplinary PhD Workshop (IIPhDW), pp. 81-84, May 2018.

[14] V. Mosorov and D. Sankowski, "Estimation of the rotation angle of gas/solid swirl flow by subpixel image resizing," Asia-Pacific Journal of Chemical Engineering, vol. 13, no. 2, p. e2177, 2018.

[15] A. Romanowski, "Big data-driven contextual processing methods for electrical capacitance tomography," IEEE Transactions on Industrial Informatics, vol. doi:10.1109/TII.2018.2855200, p. in press, 2018.

[16] A. Schmidt, "Implicit human computer interaction through context," Personal Technologies, vol. 4, no. 2, pp. 191-199, 2000.

[17] E. Pascalau, G. Nalepa, and K. Kluza, "Towards a better understanding of context-aware applications," in FedCSIS13, ACSIS, IEEE, p. 959962 , 2013.

[18] A. Romanowski, K. Grudzien, Z. Chaniecki, and P. Wozniak, "Contextual processing of ECT measurement information towards detection of process emergency states," in Hybrid Intelligent Systems (HIS), 2013 13th International Conference on, pp. 291-297, 2013.

[19] I. Jelliti, A. Romanowski, , and K. Grudzien, "Design of crowdsourcing system for analysis of gravitational flow using x-ray visualization," in FedCSIS16, ACSIS, vol. 8. IEEE, p. 16131619, 2016.

[20] C. Chen, P. W. Woźniak, A. Romanowski, M. Obaid, T. Jaworski, J. Kucharski, K. Grudzień, S. Zhao, and M. Fjeld, "Using crowdsourcing for scientific analysis of industrial tomographic images," ACM Trans. Intell. Syst. Technol., vol. 7, no. 4, pp. 52:1-52:25, 2016.

[21] M. Skuza and A. Romanowski, "Sentiment analysis of twitter data within big data distributed environment for stock prediction," in 2015 FedCSIS'15, pp. 1349-1354, Sept 2015.

[22] H. Garbaa, L. Jackowska-Strumillo, K. Grudzien, and A. Romanowski, "Neural network approach to ect inverse problem solving for estimation of gravitational solids flow," in 2014 Federated Conference on Computer Science and Information Systems, pp. 19-26, Sept 2014.

[23] R. A. Darwich and L. Babout, "Investigating local orientation methods to segment microstructure with 3d solid texture," IET Image Processing, vol. 12 , no. 7, pp. 1265-1272, 2018.

[24] S. Waktola, K. Grudzien, L. Babout, and J. Adrien, "Local concentration changes in eccentric and concentric silo discharging modes using $\mathrm{x}$ ray tomography," in 2018 International Interdisciplinary PhD Workshop (IIPhDW), pp. 377-380, May 2018.

[25] A. Wojciechowski and K. Fornalczyk, "Exponentially smoothed interactive gaze tracking method.," Springer, Cham, In International Conference on Computer Vision and Graphics (pp. 645-652), 2014.

[26] A. Wojciechowski and R. Staniucha, "Mouth features extraction for emotion classification," in FedCSIS16, ACSIS, vol. 8. IEEE, p. 16851692, IEEE, 2016. 